\title{
Guidelines for Management of Infants Delivered during the COVID-19 Pandemic in the USA and "Across the Pond"
}

Ahmed Afifi, MBBCH, MSc, David Corcoran MD, Allison C. Walker, MD, Alexandra Adamczak, M.D., T. Allen Merritt, MD, Jan Mazela, MD, PhD, Thomas A. Clarke, MD

The COVID-19 pandemic has brought unprecedented contemporary challenges to the delivery of health care to pregnant women and their infants. The World Health Organization (1), and the Centers for Disease Control and Prevention (2), and the American Academy of Pediatrics (3) have issued guidelines regarding mother-infant-postpartum care if a mother is COVID-19 positive and emphasize the importance of a model of shared decision making between mother, health care providers, and family members regarding the need for separation of mothers and infants while they are in the hospital. The guidelines from the American Academy of Pediatrics have been undergoing revision with the latest update provided on May 21, 2020. Key guidance focuses on the use of delayed-cord clamping and that "there is no reason why the infant should not have the benefits of delayed cord clamping and skin-to-skin contact after delivery." The amended guidance acknowledges that "experts are divided" regarding rooming-in for mothers. "While difficult to separate mother and infant, this is the safest action, at least temporarily," as they may provide the mother time to become less infectious," and the goal is not to separate a family from its newborn." The American Academy of Pediatrics strongly supports breastfeeding as "to date, breast milk is considered to be an unlikely course of transmission of SARSCoV-2 and encourages mothers who are COVID-19 positive to express breastmilk after appropriate hygiene which may be fed to the infant by an un-infected caregiver. If mothers prefer to nurse their infant, they should follow strict preventive precautions. Early hospital discharge is discouraged, and frequent post-discharge follow is recommended. Guidance for visitation to the NICU is that mothers and partners who are COVID-10 positive or persons under investigation should not enter the NICU until their status is resolved. (www.aapnews.org May 21, 2020).

The initial Chinese response and guidance regarding neonatal management during the Coronavirus (COVID-19) outbreak was reported by $\mathrm{Ma}$ and coworkers from Wuhan, China (4), in May 2020. In the April edition, Neonatology Today featured management guidelines for the SARS-CoV-2 virus in Ontario, Canada (5). However, there are some important differences in these guidelines, and there has been some "push back" from some groups regarding the duration of maternal-infant separation, restrictions on mother's caring for their infant, including breastfeeding, or the exclusive use of expressed breast milk rather than breastfeeding using some personal protective equipment (PPE). Obstetricians, midwives, nurses, neonatologists and paediatricians attending deliveries are performing procedures that are associated with aerosolization of infant respiratory secretions and maternal blood and/Or vaginal secretions that pose hazards to those providing care for mothers and their infants. Furthermore, suggested changes in "Neonatal Resuscitation Where the Mother Has a Suspected or Confirmed case of COVID-19" in terms of neonatologist use of personal protective equipment and safety for healthcare workers and management of mothers in Italy were published by Trevisanuto et al. (6) and $\mathrm{Ng}$ et al. in Hong Kong (7). The American Heart Association and the American Association of Critical Care Nurses have recently published interim guidelines regarding neonatal resuscitation and support of children and neonates with suspected or confirmed COVID-19 (8)

\section{"The American Heart Association and the American Association of Critical Care Nurses have recently published interim guidelines regarding neonatal resuscitation and support of children and neonates with suspected or confirmed} COVID-19 (8)"

Current data support the limited vertical transmission of COVID-19 via the placenta with the greatest risk associated with maternal blood and aerosolized secretions occurring at the time of birth or immediately afterward. Two recent reports from China found no clinical findings or investigations suggestive of COVID 19 in neonates born to affected mothers, and all samples, including amniotic fluid, cord blood, and breast milk, were negative for SARS-CoV-2, the vertical maternal-fetal transmission cannot be ruled out. $(6,7)$ Zeng et al. reported that 3 of 33 infants presented with early-onset SARS-COV-2 infection even when strict infection control measures were taken during delivery. The authors conclude that the likely sources of the infant's infection were of maternal origin (9).

In the Republic of Ireland had 25, 163 confirmed cases of COVID-19 with 1670 deaths (6.6\%), Northern Ireland 4776 cases with 535 deaths $(11.2 \%)$ while in Poland 25410 cases and 1137 deaths (4.4\%) reported as of June 5, 2020 (https//www.statista.com/statistics/1043366/novel-coronavirsu-2019nvov-casesworldwidebycounty.html). Other countries in Europe face similar challenges with the exponential rise of cases in Italy, Spain and Germany during April 2020. Neonatology Today, co-editors in Ireland and Poland have shared their guidelines for the management of infants in Ireland, Belfast, Northern Ireland, and Poland during this worldwide pandemic.

\section{Ireland}

Ireland has 19 maternity hospital departments and nurseries, and in Ireland, the Institute of Obstetricians and Gynaecologists of the Royal College of Physicians of Ireland outlined recommendations for Neonatal Management for Maternal and Neonatal Manage-

NEONATOLOGY TODAY is interested in publishing manuscripts from Neonatologists, Fellows, NNPs and those involved in caring for neonates on case studies, research results, hospital news, meeting announcements, and other pertinent topics.

Please submit your manuscript to: LomaLindaPublishingCompany@gmail.com 
ment (11). These guidelines for Neonatal Management are summarized as steps to be taken at Delivery and Policies in the Neonatal Nursery Unit, and Discharge Home. These are summarized as follows (please note spellings conform with this guideline).

Delivery: The neonatal team should be informed of plans to deliver the baby of a woman affected by COVID-19 infection, as far in advance as possible and should also be given sufficient notice at the time of birth, to allow them to attend and don PPE before entering the room/theatre. However, COVID-19 infection in the mother is not per se an indication for the neonatal team to routinely attend low-risk delivery. Furthermore, there is a risk that medical staff attending such deliveries may be more difficult or have delayed response time to concurrent emergencies.

A designated member of the neonatal team should be assigned to attend suspected/confirmed COVID-19 deliveries. The most senior person likely to be required must attend in the first instance to minimize staff exposure. If there is a high rate of maternal infection, units might choose to establish a dedicated COVID Neonatal Team with a dedicated Registrar and Consultant during working hours. Local units should make their own arrangements for designating staff, but senior involvement is expected. PPE should be donned in an adjacent room, and the team member should wait outside the delivery room, ready to be called in should the baby, require any intervention(s). If it is anticipated that the baby will require respiratory support, appropriately skilled neonatal team members should be present at delivery wearing PPE.

Neonatal resuscitation/stabilization should proceed as per guidance. If additional equipment is required, this can be passed to the team by a "clean" staff member outside the room. Neonates should be transferred in a closed incubator, although where the baby is unwell, they may need to be transferred by resuscitaire or resuscitation warmer (with staff in full personal protective equipment). Where possible, all procedures and investigations should be carried out in a single room or an isolation room/bay with a minimal number of staff present.

There is no evidence to suggest that antenatal corticosteroids for fetal lung maturation cause any harm in the context of COVID-19, except perhaps where the pregnant woman has a critical illness in which case a multidisciplinary discussion is required to determine their relative benefits. Steroids should, therefore, be given to mothers anticipating preterm delivery where indicated, and urgent delivery should not be delayed for their administration. Magnesium Sulphate should be given for neuroprotection of babies $<32$ weeks' gestation as per the current guidance.

Regarding neonatal management of suspected, probable, and confirmed cases of maternal COVID-19 infection, the umbilical cord should be clamped, and the neonate should be transferred to the resuscitation area for routine assessment and if appropriate assessment by the attending neonatal team. There is insufficient evidence regarding whether delayed cord clamping (DCC) increases the risk of infection to the newborn via direct contact. The most recent guidance clearly states that deferred cord clamping is still recommended, provided there are no other contraindications. The baby can be dried as normal while the cord is still intact. In the case of a preterm baby, standard thermoregulatory measure, including the use of a plastic bag, should also be used.

Whether DCC is practiced or not, the neonate should be transferred after delivery to the resuscitaire for initial assessment by the attending midwife, or by the neonatal team as appropriate. An Immediate skin to skin approach with the COVID-19 positive mothers should not take place; this can be considered when the mother has taken appropriate hand hygiene and sterile PPE precautions. Asymptomatic well babies should not be admitted to the neonatal unit. Babies of COVID-19 positive mothers who need admission to the neonatal unit (NNU) for any reason should be isolated and managed in their own isolette in a designated isolation area, with dedicated staffing.

\section{Rooming-in and Infant Feeding}

Given the current lack of information, it seems reasonable to assume that a newborn from a mother with COVID-19 at delivery could possibly be infected, either in utero or perinatally, and thus should be placed in isolation to avoid exposure to other newborns. However, well term/near-term babies, not otherwise requiring neonatal unit care should stay with their mother, if at all possible. If the mother is severely or critically ill, separation may then be necessary, but the need for this should be regularly reviewed. Maternal illness is not in itself an indication for newborn admission to the NNU so that the baby may be cared for in an isolette in the nursery or isolation with the mother, e.g., on a COVID-19 assigned ward. It is recommended that the baby is cared for at home if the mother is admitted to an Acute Adult Hospital to reduce the risk of infection to the baby. In light of current evidence, the benefits of breastfeeding outweigh any potential risks of transmission of the virus through breastmilk. If the woman is asymptomatic or mildly affected, breastfeeding should be supported by encouraging mothers in coordination with healthcare providers. Breastfeeding can still be supported by encouraging mothers who have been separated from their babies to express milk. Either way, mothers should have a designated breast pump for exclusive use and local infection being followed regarding equipment decontamination.

\section{"Breastfeeding can still be supported by encouraging mothers who have been separated from their babies to express milk. Either way, mothers should have a designated breast pump for exclusive use and local infection being followed regarding equipment decontamination."}

In the case of rooming-in, the baby's cot should be kept at least 2 meters from the mother's bed, and a physical barrier such as a curtain may be used. An incubator can also be used in the room as a physical barrier. Babies requiring subsequent additional care (e.g., intravenous antibiotics) should be assessed in the delivery suite or postnatal wards and a decision made as to whether additional care can safely be provided at the mother's bedside. NNU admission should be avoided if at all possible and safe. Any need to separate mothers with COVID-19 infection from their newborns, with the consequence that they are unable to breastfeed directly, may impede early bonding as well as the establishment of lactation. These factors will inevitably cause additional stress for mothers in the postpartum period. As well as caring for their physical wellbeing, medical teams should consider the mental wellbeing of these mothers, showing appropriate concern and providing support when needed.

The Neonatal Paediatric COVID-19 guidance group have issued recommendations for breastfeeding during the COVID-19 pandemic. These state that the Faculty of Paediatrics encourage breastfeeding to protect children and reiterate that "the benefits of breastfeeding outweigh the potential for exposure to the virus.

\section{Testing:}


There is currently no clinical indication to test any well-baby born to a COVID-19 positive mother. Performing nasal swabs on asymptomatic infants may also result in false negatives, and the optimal time of testing, in any case, is unclear. Asymptomatic patients, including infants, even if positive, are unlikely to transmit the virus, providing everyone adheres to basic hygiene measures. Viral RNA may be detectable in the stool for several weeks, but this does not mean that the faecal material is necessarily infective; providing caregivers adhere to the basic hygiene measures, the risk is not thought to be significant.

Asymptomatic babies should not be routinely admitted to the NNU. If subsequently admitted for other issues such as jaundice or hypoglycaemia they do not require testing unless their symptoms fit the case definition. Case definition: newborns may not show all the features of influenza-like illness, particularly a fever, so clinicians should have a high index of suspicion in all babies admitted to the NNU, and monitor for signs of respiratory illness during the admission.

Babies of COVID-19 positive mothers who need admission to the $\mathrm{NNU}$ for any reason should be isolated and managed in their own isolette in a designated isolation area, with dedicated staffing. They must be monitored for signs of COVID-19 during their admission. If they develop signs, they should be tested. Symptomatic babies that meet the definition only by virtue of requiring respiratory support for an anticipated non-COVID-19 respiratory pathology (e.g., RDS) should be tested after 72 hours of age to avoid potential early false-negative results. It is suggested to test again on day five before declaring them negative and non-infected.

Babies can come out of isolation despite continuing to need for respiratory support, providing the tests on days 3 and 5 are negative, and the baby is following the projected clinical course (e.g., expected for RDS, etc.). If there is clinical concern that a baby who has been in isolation meets the case definition is not following a typical clinical course for an anticipated non-COVID-19 respiratory pathology, they should be tested that day.

Known COVID-19 positive babies should be isolated until their symptoms resolve, and they no longer need respiratory support; they can then be allowed out of isolation but must remain in an incubator and monitored respiratory signs and symptoms for a further 14 days. Babies awaiting test results and less than seven days of age can be cohorted in the same isolation room, provided they remain in incubators; airborne transmission (except for aerosol-generating procedures) is not currently thought to be a major mechanism of transmission in this clinical context.

Clinical investigations should be minimized whilst maintaining standards of care. In the absence of evidence, it is reasonable to treat the baby's respiratory illness in the same way as if they were not potentially exposed to COVID-19. The evidence in favour of early intubation is limited to adults and older children. All babies requiring respiratory support should be nursed in an incubator. Intubation is an aerosol-generating procedure, although the risk of transmission soon after birth is thought to be low; however, it is recommended that staff follow their local guidelines regarding the use of appropriate PPE, even in an emergency. In-line suction with an endotracheal tube should be used where possible. Where possible, the use of a video-laryngoscope should be considered for intubation, which might facilitate keeping the baby within the incubator. By reducing proximity to the baby's airway, this may help to reduced exposure to the virus. Intubation should be undertaken by staff with appropriate competencies. CPAP and high flow therapies are associated with aerosolization, and staff caring for infants receiving these therapies must also adhere to their local guidance regarding the use of appropriate PPE.
Transfers should be limited to a minimum, and per network escalation policies. Exposure to COVID-19, in itself, is not a reason to transfer. All staff must adhere to the locally recommended PPE guidelines before entering the isolation room. A register must be kept of all staff entering isolation rooms. All equipment coming out of the isolation room should be cleaned. It is anticipated that NNU capacity may become problematic either due to cot capacity or staff availability. Individual units should have agreed to staffing plans when optimal staffing plans cannot be achieved. The cohorting of confirmed positive cases may be necessary and should follow local guidance.

COVID-19 positive mothers should not visit their baby on the NNU until they are asymptomatic and have tested negative. Partners of COVID-19 positive mothers must adhere to the current advise regarding self-isolation and the hospital policy regarding visiting the maternity wards and NNU, except under exceptional circumstances.

\section{Newborn Screening:}

Newborn Infant physical examination should be completed as usual in the hospital, prior to discharge. Newborn Blood Spot screen should take place as usual. Audiology screening should continue in maternity units and on the NNU. The ability to perform investigations and tests once the infant has left hospital will be restricted, and when possible, tests should be performed before discharge from the maternity or neonatal units. Maternity units should aim to maintain sufficient staffing in order to perform the necessary screening before discharge.

\section{Discharge Home:}

When babies and mothers are ready for hospital discharge, they should be provided with written advice regarding what to look out for in terms of respiratory symptoms, lethargy or poor feeding, and from whom to seek further advice should they have concerns. They should be advised to self-isolate for 14 days. All measures aimed at early discharge from the NNU should be scaled up, where possible, to avoid vulnerable infants with chronic lung disease attending clinics. Consider telephone/video consultation for neonatal follow up when possible, to avoid vulnerable infants from attending clinics.

"Given the current lack of information, it seems reasonable to assume that a newborn from a mother with COVID-19 at delivery could possibly be infected, either in utero or perinatally, and thus should be placed in isolation to avoid exposure to other newborns. However, well term/ near-term babies, not otherwise requiring neonatal unit care should stay with their mother, if at all possible. "

Advice should be provided to parents of those infants at increased risk (e.g., immunocompromised, chronic lung disease, cardiac disease) about reducing the risk of infection (reduced social contact, handwashing) and interventions aimed at preventing other diseases (e.g., immunizations) should be optimized. Parents who 
telephone NNUs for help should receive experienced advice, with the aim of minimizing direct contact with either neonatal or paediatric services.

Among the 19 maternity hospitals and nurseries, or among midwives delivering at home (which is rare in Ireland) there has been a survey or an audit of adherence to these recommended practices; nonetheless, there is great hope that having a standardized approach will reduce maternal to infant transmission of COVID-19 and protect professionals caring for both mothers and their babies."

Northern Ireland

At the Royal Maternity Hospital in Belfast, Northern Ireland, policies were developed in response to COVID-19, focusing on specific guidance around infection prevention within the National Health Service of the United Kingdom. (12)

Hand Hygiene: Strict hand hygiene must be adhered to. Arms should be bare to the elbows, with no hand and wrist jewelry except for a single metal ring band), have clean short fingernails with no artificial nails or nail products, and any cuts or abrasions should be covered with waterproof dressings. The Seven-Step Technique should always be observed when washing or gelling hands.

Uniform: Staff are asked to travel to and from work in their own clothes and change into scrubs once on site. Scrubs should then be removed at the end of a shift and placed in the appropriate laundry bag. There are facilities available if staff wish to shower before traveling home.

Social Distancing: Where possible, staff are asked to maintain a distance of 2 metres between each other while at work. Additional alternative areas for breaks will be made available. Measures such as limiting the number of infants within a room, and limiting one parent to the bedside at a time are also being undertaken to facilitate this.

Essential Personnel Only: Only essential personnel should physically attend work. Where possible staff should try and facilitate working from home via remote access, video conferencing, and other methods of remote access. Elective and non-urgent work should be reviewed and either canceled or relocated as able.

Aerosol Generating Procedures requires additional care when performed on patients with suspected or confirmed COVID-19. Aerosol Generating Procedures should only be carried out when absolutely necessary, with only essential staff present. These include:

Intubation, extubation, and related procedures, manual ventilation, Less invasive surfactant administration, Open suctioning, non-invasive ventilation, e.g., Bi-level positive airway pressure (BiPAP and Continuous Positive Airway Pressure Ventilation (CPAP), high-frequency oscillating ventilation, High flow Nasal Oxygen, Tracheotomy/tracheostomy procedures. Placement of an oral or nasal gastric tube, use of low flow oxygen, nebulisers and Entonox are considered to be aerosol-generating procedures.

Personal Protective Equipment. On April 4, 2020, Public Health England recommend that due to sustained background transmission of COVID-19 within the UK, personal protective equipment is required in every patient area regardless of infection status. This led the Belfast Trust to categorise all hospital areas with a traffic light "zoning" system. This has been modified with the Neonatal Nursery Unit as follows:

Green Zone: Areas that are generally considered "clean" with no direct patient contact, i.e., main corridors, tea room, canteen, and offices where NO PPE are required

Amber Zone: These areas will contain infants, who may be undergoing aerosol-generating procedures, but have had no exposure to coronavirus and consist of the main clinical areas with the NICU and main delivery suite.-Standard PPE=Apron and non-sterile gloves, fluid-resistant surgical face mask, and the use of eye protection should be considered if risks of splashes or droplets.

Red Zone: These areas will contain infants of mothers with suspected or confirmed COVID-19 or infants who are at risk of horizontal transmission where enhanced PPE required. Attendance at all deliveries of suspected or confirmed COVID-19 positive mothers, working within a COVID-19 cohort area with infants with suspected or confirmed COVID-19 virus, where aerosol-generating procedures are being performed or anticipated, and when resuscitating the acutely collapsed infant regardless of maternal status, will also require advanced PPE. This enhanced PPE includes FFP3 respiratory mask, long-sleeved disposable gown, disposable eye protection, Gloves, Apron, disposable hat. All PPE must be donned and doffed in an established sequence, with specific care and attention taken at each stage. Donning PPE with a "buddy" ensures satisfactory technique and enhances user safety.

Antenatal Admission and Delivery at Royal Maternity Hospital (RMH), Belfast

Entry to $\mathrm{RMH}$ : Everyone visiting or presenting to the $\mathrm{RMH}$ will now have to buzz for entry to the building with strict entry criteria in place due to the restricted visiting policies. If entry is permitted, the person will be allowed access to the reception area before being asked a series of questions including:

Do you have a cough?

Do you have a temperature?

Do you have any shortness of breath?

Are you/have you recently been, self-isolating?

"All PPE must be donned and doffed in an established sequence, with specific care and attention taken at each stage. Donning PPE with a "buddy" ensures satisfactory technique and enhances user safety."

\section{Have you been in contact with anyone who is self-isolating or had COVID19?}

If the answer to any of these is 'yes' the person will either be denied entry or treated as a patient with 'suspected COVID-19'. For the purpose of this guideline, we are defining a mother with 'suspected COVID' as a person who has had swabs taken, but results are not yet available.

Any woman presenting to admission with either suspected on confirmed COVID-19 will be transferred to an active birthing centre room. The donning area for these rooms is set up in the corridor just outside these rooms. The doffing areas are in the corridors between rooms. A dedicated COVID-19 resuscitation trolley 
Antenatal admission of woman with suspected

or confirmed COVID-19

$<27$ weeks gestation
-Neonatal team to be informed at time of admission

- Senior neonatal team to meet and begin preparations including: identification and allocation of staff roles location of mother route for transfer of infant intended admission location for infant

\section{Delivery, resuscitation and stabilisation}

\section{- Designated consultant and senior nurse to attend delivery}

-Attend delivery area and don appropriate PPE, in designated donning area

- Only when delivery is anticipated, enter delivery area and prepare resuscitaire and essential equipment

-Delayed cord clamping should be considered as per unit protocol

- Infant should be moved directly to resuscitaire once cord cut

- Resuscitate and stabilise as per NLS algorithm

-An in-line micro HME filter should be used with all respiratory support
Maternal suspected or confirmed COVID-19: mother asymptomatic or only mildly symptomatic
-Infant may be intially shown to parents but social distancing should be observed

- Once infant stabilised, if the mother is able to apply a surgical face mask and gel hands, she may have contact with her infant if desired and/or feasible, prior to transfer to NICU
Maternal suspected or confirmed COVID-19, mother symptomatic and/or acutely unwell
-Infant may be shown to parents but social distancing should be observed throughout 


\section{Transfer to NICU}

- Once the infant has been stabilised, NICU should be informed of the pending admission

- Transfer to NICU should be via the agreed route only

-Additional 'clean' helpers should be available to clear corriders, and open doors etc

- Only the 'middle lift' should be used for transport between floors

-Infants $<27$ weeks gestation should be transferred using a resuscitaire

-Admit to designated cohort area within NICU

- Give a clear handover to the receiving team before transferring the infant to the incubator

- The transport resuscitaire should be moved to the designated doffing area to have an initial clean, before moving it to an area outwith the cohort area for further cleaning

- Staff should doff PPE in the designated area before exiting the cohort area

\section{Inform NNNI}

-The NNNI should be informed of all admissions of an infant born to a mother with suspected or confirmed COVID-19

- Consideration should be made to whether a Network Call should be scheduled 


\section{7 - 35 Weeks Gestation}

Antenatal admission of woman with suspected or confirmed COVID-19 27 - 34+6 wks gestation
- Neonatal team to be informed at time of admission

- Senior neonatal team to meet and begin preparations including: identification and allocation of staff roles location of mother route for transfer of infant intended admission location for infant
Delivery, resuscitation and stabilisation
-ST4+ and senior nurse to attend delivery unless infant considered 'high risk'

-Attend delivery area and don appropriate PPE, in designated donning area

- Only when delivery is anticipated, enter delivery area and prepare resuscitaire and essential equipment

- Delayed cord clamping should be consdiered as per unit protocol

- Infant should be moved directly to resuscitaire once cord cut

- Resuscitate and stabilise as per NLS protocols

-An in-line micro HME filter should be used with all respiratory support

-All infants of this gestation will require admission
Maternal suspected or confirmed COVID-19: mother asymptomatic

or

only mildly symptomatic
- Infant may be intially shown to parents but social distancing should be observed

- Once infant stabilised, if the mother is able to apply a surgical face mask and gel hands, she may have contact with her infant if desired and/or feasible, prior to transfer to NICU
Maternal suspected or confirmed COVID-19, mother symptomatic and/or acutely unwell
- Infant should be shown to parents but social distancing should be observed throughout 


\section{Transfer to NICU}

- Once the infant has been stabilised, inform the NICU of the pending admission

- Transfer to NICU should be via the agreed route only

- Additional 'clean' helpers should be available to clear corriders, and open doors etc

- Only the 'middle lift' should be used for transport between floors

- Infants requiring respiratory support should be transported on a resuscitaire. All other infants may be transported in an incubator

- Admit to designated cohort area within NICU

- Give a clear handover to the receiving team before transferring the infant to the incubator

- The transport resuscitaire or incubator should be moved to the designated doffing area to have an initial clean, before moving it to an area outwith the cohort area for further cleaning

- Staff should doff PPE in the designated area before exiting the cohort area

- The NNNI should be informed of all admissions of an infant born to a mother with suspected or confirmed COVID-19

Inform NNNI

- Consideration should be made to whether a Network Call should be scheduled 


\section{Greater Than or Equal to 35 Weeks Gestation}

Antenatal admission of woman with suspected or confirmed COVID-19 $\geq 35$ weeks gestation
- Neonatal team to be informed at time of admission - Senior neonatal team to meet and begin preparations including: identification and allocation of staff roles location of patient route for transfer of infant

\section{Attendance at delivery}

\section{Delivery, resuscitation} and stabilisation
- Neonatal attendance at deliveries of infants $\geq 35$ weeks should be requested as per current RMH policy

- Suspected or confirmed maternal COVID-19 status is NOT an indication in itself for neonatal attendance at birth

\section{- If neonatal attendance is required at delivery but the}

is infant considered 'low risk' - ST4+ to attend delivery

- If infant considered 'high risk' - consultant and senior nurse to attend and be present in room prior to delivery

-Attend delivery area and don appropriate PPE in designated donning area

-Wait outside delivery area and only enter room if newborn requires resuscitation (unless high risk). It is the responsibility of the midwifery team within the delivery area to have checked and prepared resuscitaire

-Delayed cord clamping should be performed as per unit protocol

- If required, resuscitate and stabilise as per NLS algorithm

-Use an in-line micro HME filter filter for all respiratory support

- If infant well, every effort should be made to keep the infant and mother together postnatally

Maternal suspected or confirmed COVID-19: mother asymptomatic

or

only mildy symptomatic
- Depending on maternal infection status and symptoms, there should be consideration of maternal use of a face mask when handling and feeding the infant while in hospital, with distancing measures observed at other times. Infant should be nursed in an incubator

-Discharge home should be facilitated as soon as feasible

- Family should be educated in hygiene and distancing measures to avoid viral spread 


\section{Maternal confirmed COVID-19:}

mother acutely unwell

\section{Transfer to NICU}

\section{Admission to NICU}

-Infant to be isolated from mother at birth. If the infant is well it may remain within same room initially, within an incubator, until a suitable carer or care area can be identified (not NNU)

- Infant discharge with an alternative carer (clinically well and not self isolating) should be considered

- If the infant requires admission, NICU should be urgently informed of the pending admission

- Transfer to NICU via the agreed route only

- Additional 'clean' helpers should be available to clear corriders, and open doors etc

- Only the 'middle lift' should be used for transport between floors

- Infants requiring respiratory support should be transported on a resuscitaire. All other infants may be transported in an incubator
-Admit to designated cohort area within NICU

- Give a clear handover to the receiving team before transferring the infant to the incubator

- The transport resuscitaire or incubator should be moved to the designated doffing area to have an initial clean, before moving it to an area outwith the cohort area for further cleaning

- Staff should doff PPE in the designated area before exiting the cohort area

\section{Inform NNNI}

-The NNNI should be informed of all admissions of an infant born to a mother with suspected / confirmed COVID-19

- Consideration should be made to whether a Network Call should be scheduled 


\section{Resuscitation Equipment}

Newborn resuscitation should continue as per the standard NLS algorithm.

Although the vertical transmission of SARS-CoV-2 is considered possible, it remains to be definitively proven. It is assumed that, even if an infant was infected at birth, the viral load would be either very low or undetectable. This, in combination with the fact that infants' lungs are not aerated at time birth and much lower tidal volumes are used compared to adults practice, means that newborn resuscitation, including AGPs, is considered to carry a low risk of infection.

However, we are advising a slight alteration to our standard equipment to further minimise risk of transmission to staff. This is the inclusion of an in-line heat and moisture exchanger (HME) micro filter during respiratory support. These filters should be used for both Neopuff/mask support and when intubated. The photos below demonstrate their position within the equipment. Although some recent work has indicated that tidal volumes and pressures delivered are not affected with the inclusion of a filter, both the weight and the potential dead space of the circuit may be increased and staff should be cognisant of this. Once the ETT position is confirmed with the Neostat and visible chest rise, there is an option to remove the Neostat from your circuit.

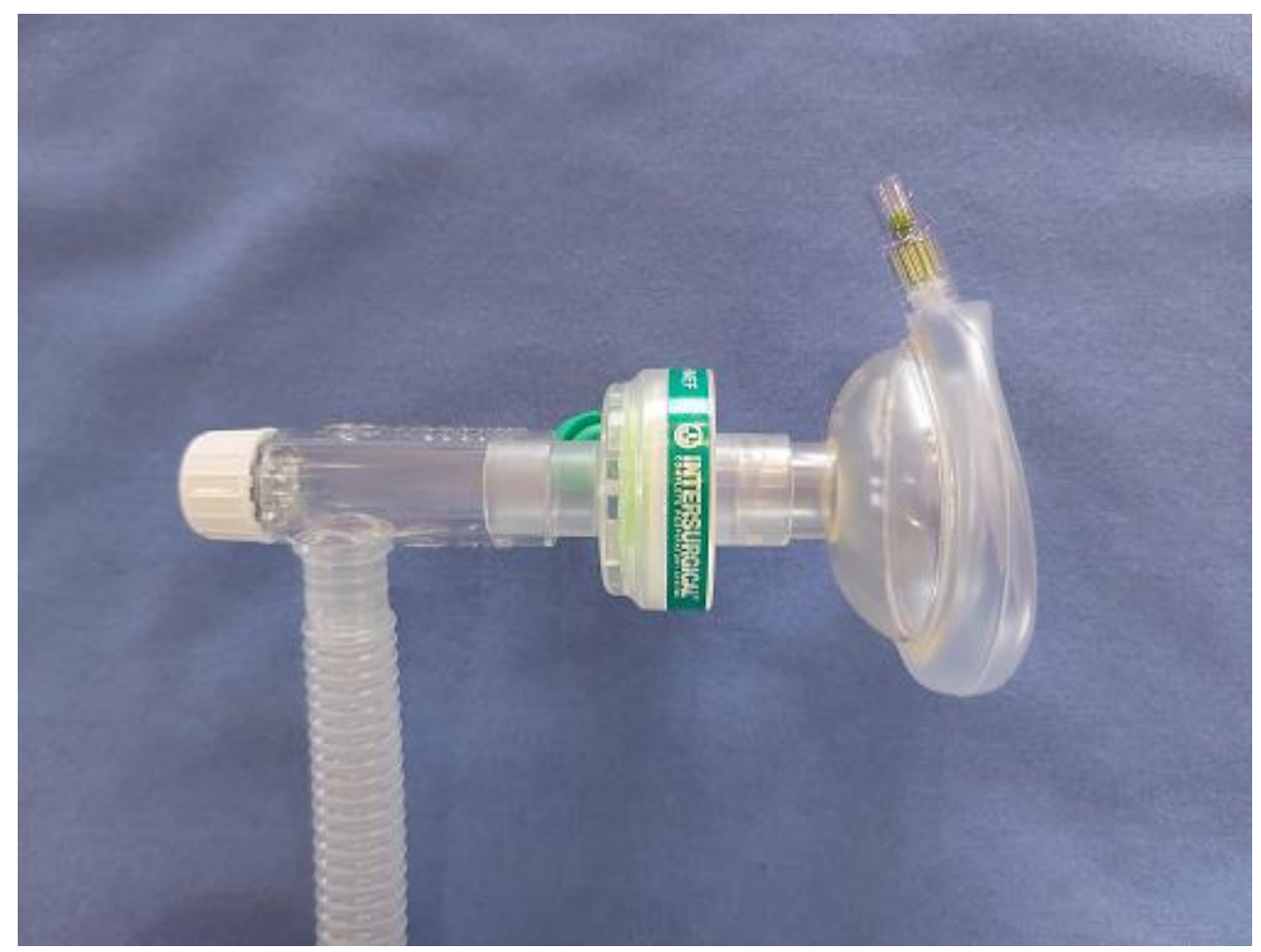

Neopuff and Mask with In-Line HME Filter 


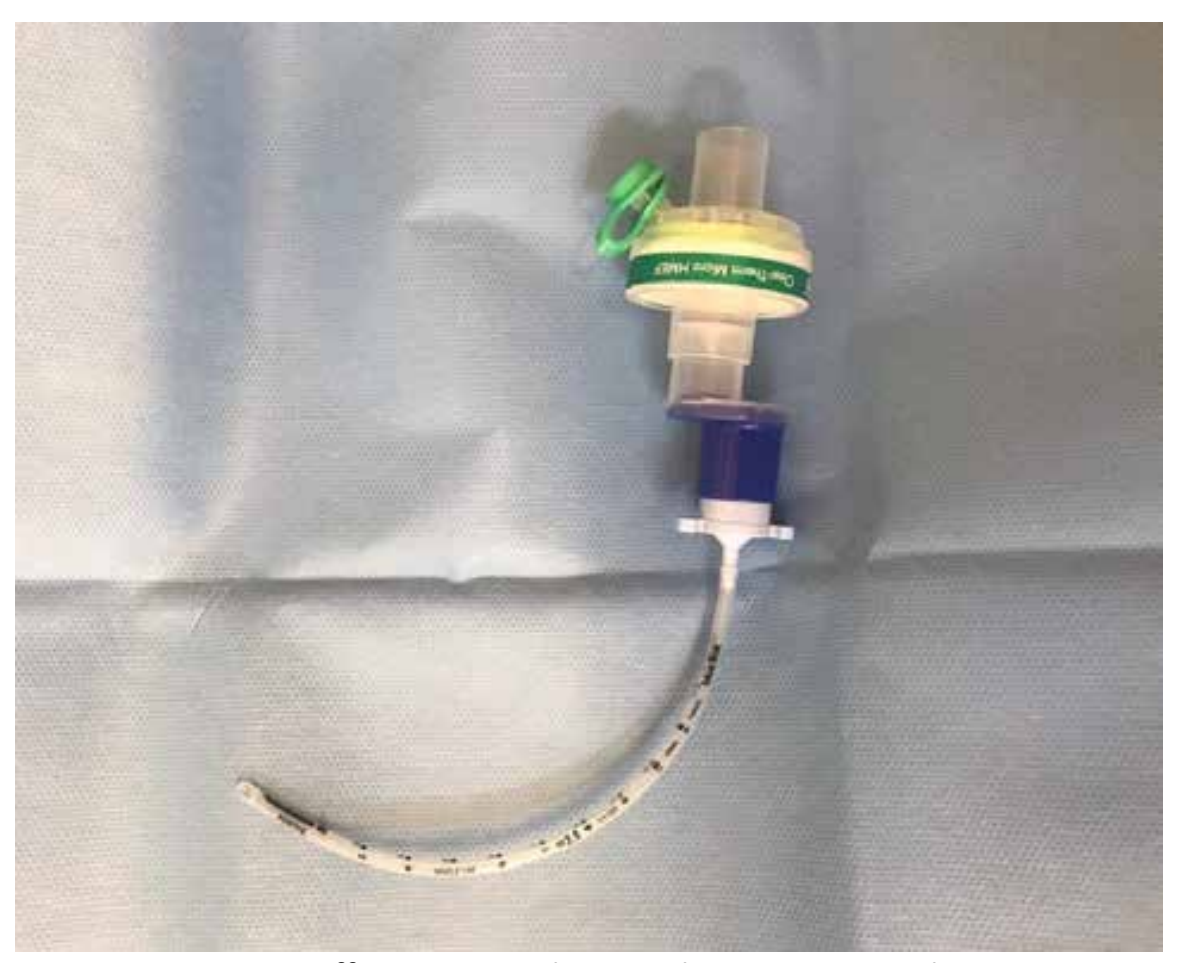

Neopuff, Neostat and ETT with In-Line HME Filter

\section{Transfer of a Newborn Infant to NNU}

If a newborn requires admission to the NNU at birth, the Sister-in-charge should be informed as soon as this decision is made in order to allow time for preparation and staff allocation.

The infant will be admitted to the cohort area within the NNU.

There are specified transfer routes out of both delivery suite and theatres and these should be adhered to. Specifically, the potentially exposed team and infant should NOT use the back lift or enter the NNU through the NICU area.

Additional identified members of staff will travel before and behind the neonatal transfer team to ensure corridors are cleared and doors are open. They will remain at a distance of at least 2 metres from the team and will not assist in the transport itself.

From Delivery Suite (Rooms 19 - 16): Exit out of the back door of the link corridor (doffing area) onto the main hospital corridor. Travel to the middle lift and ascend to the $2^{\text {nd }}$ floor. Enter the NNU cohort area via the side door.

From Theatre (ground floor): Exit out of Theatre 2 onto the main theatre corridor. Travel out through theatre reception into the main corridor and out onto the main hospital ground floor. Travel to the middle lift and ascent to the $2^{\text {nd }}$ floor. Enter the NNU cohort area via the side door.

Walk through videos of each of these routes have been made and shared with the wider group. 


\section{Admission to NNU: Suspected Newborns}

All preterm or term unwell infants of mothers with either suspected or confirmed COVID-19 will be admitted directly to the cohort area in the NNU (see below). Given the low likelihood that a newborn will be COVID-19 positive at birth, medical treatment and management should be mainly determined based on their pathology and clinical needs rather than being influenced by specific considerations around coronavirus.

All infants should be nursed in a closed incubator for the duration of their stay within the cohort area. This acts as a further layer of isolation.

\section{COVID-19 Screening in Infants of Suspected or Confirmed Mothers}

All infants of mothers who have a suspected or confirmed COVID-19 status, admitted to the NNU immediately following birth, require screening. It has been suggested that optimal testing for possible vertical transmission should include IgM / IgG analysis of cord blood at birth. This requires written parental consent but is not being undertaken in $\mathrm{RMH}$ until reliable IgM/IgG testing has been developed.

Current screening schedule for infants admitted at birth is:

- $\quad$ First set of swabs at admission

- $\quad$ Second set of swabs at 72hrs (day 3)

- $\quad$ Third set of swabs on day 5

Two dry swabs should be taken at each screen with one swab of the nasopharynx and one deep throat swab. This should make the patient gag to be effective. If the infant is ventilated, then secretions obtained by ET suction should be sent. The process for swabbing is outlined below with further Trust guidance available on the Hub:

- Label the universal container before entering the cohort area. A hazard warning label should be affixed to the container

- Do not take the paper request form into the cohort area. A hazard label should also be added to the form

- Take the nasopharyngeal and throat swabs as above

- Place both swabs into the same universal container

- Wipe outside of sample with Difficil-S or Actichlor Plus (1/1000ppm)

- Place into a leak proof bag and wipe outside with with Difficil-S or Actichlor Plus

- Ideally a buddy should meet you at door and hold open a second bag for you to place leak proof bag into

- Place the form into this bag too and seal

- A 'COVID-19 biohazard' label should be attached to the outside of the second bag

- The bag containing the samples must be hand transported to the lab by a porter. The pneumatic tube system must not be used 


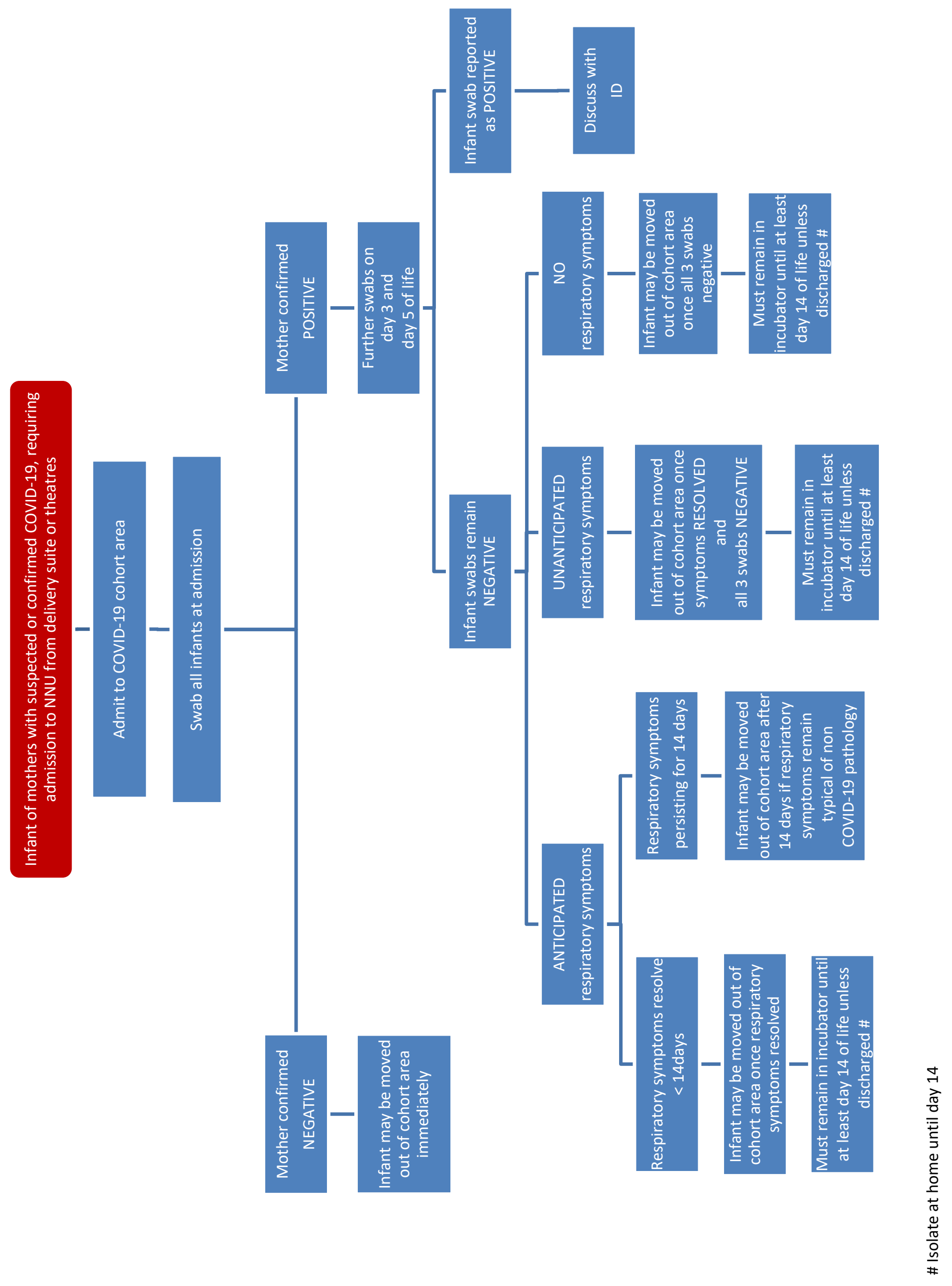




\section{Transfer Out of the COVID-19 Cohort Area}

The flow chart above also outlines timeframes for when the infant may be considered for transfer out of the cohort area.

If an infant is admitted to the cohort area due to maternal 'suspected' status, and maternal results are subsequently reported as negative, the infant may be moved out of the cohort area immediately. There is no need for a further period of isolation within an incubator, and normal neonatal care should continue.

For infants of mothers with confirmed COVID-19, the duration within the cohort area is mainly based on the presence and nature of their respiratory symptoms.

No Respiratory Symptoms: If the infant has been admitted for reasons other than respiratory support, and they have no respiratory symptoms, they may be transferred into the general clinical areas once all 3 sets of swabs are reported as negative. Examples would include late preterm infants who are admitted due to gestation and feeding support but require no respiratory support.

Anticipated Respiratory Symptoms: Anticipated respiratory symptoms are defined as clinical features in keeping with the diagnosed pathology. Examples would include respiratory distress and $\mathrm{x}$-ray changes in keeping with surfactant deficiency in a 25 week preterm infant.

Unanticipated Respiratory Symptoms: Unanticipated respiratory symptoms are defined as clinical features that are outwith the expected clinical course for an infant of their gestation or pathology. An example would be a 36 week infant with no antenatal concerns who required intubation due to increased work of breathing and climbing oxygen requirements, with no acute pathology to account for this.

If an infant is able to be moved out of the cohort area, into either the general clinical areas within the NNU or the PNW, before 2 weeks of age they should remain within an incubator until day 14 of life regardless of weight etc.

Finally, if an infant is ready for discharge home prior to a full set of swab results being performed, they may be discharged home directly from the cohort area. No further swabs will be required. The family should be advised to self-isolate at home until the infant is 2 weeks of age. 


\section{Admission to NNU: PNW Infant}

Whilst COVID-19 should be considered as a possible diagnosis in PNW infants who become unwell, it is anticipated that the majority of admissions will be more likely due to common pathologies such as hypoglycaemia or infants requiring lumbar puncture. Accordingly, the vast majority of these infants will be able to be admitted to the NNU as normal, rather than the COVID-19 cohort area.

It is expected that all cases will be discussed with the consultant on duty, regardless of symptoms. As shown in the flowchart below, an infant admitted from PNW with unanticipated respiratory symptoms, or whose mother is suspected or confirmed Covid, should be admitted directly to the COVID-19 cohort areas (Bay 3 or 4 ) initially. These infants should have swabs performed at admission with 2 more sets performed at 48 hrly intervals.

In the situation where the mother has no suspicions of COVID-19 but her infant is swabbed, the mother must also be swabbed regardless of symptoms and is unable to visit the NNU until her results are confirmed negative.

If an infant is considered fit for discharge either back to the PNW or home, prior to completing a full set of screening swabs, they should be discharged and no further swabs are required. If an infant has been investigated for coronavirus, the family should self-isolate for 14 days after the onset of symptoms, regardless of swab results.

The flow chart below aims to represent the journey of a PNW admission. We have endeavoured to represent the range of reasons for PNW admissions and try and encompass the various clinical outcomes, but recognise the complexity of the chart as a result. 


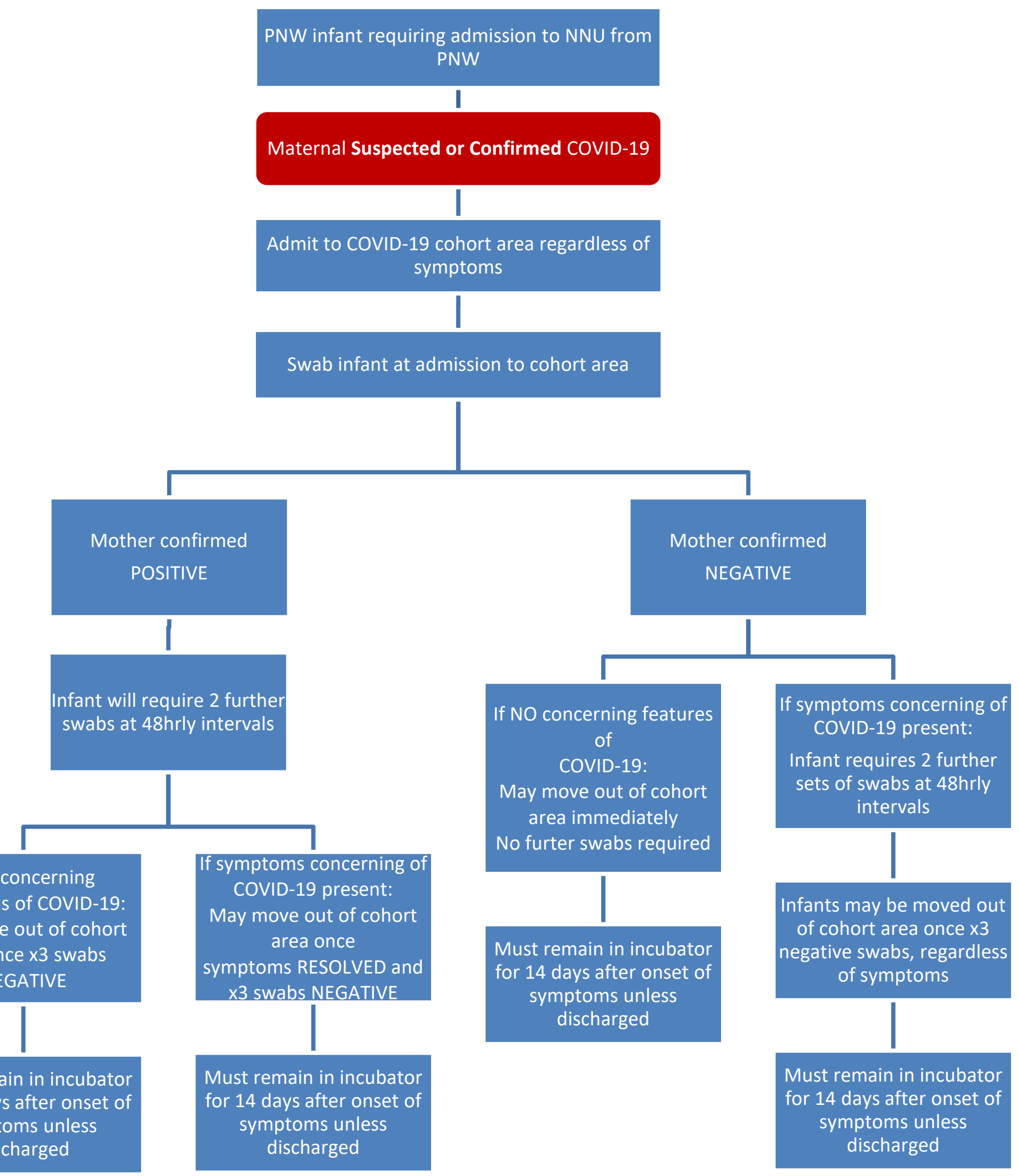




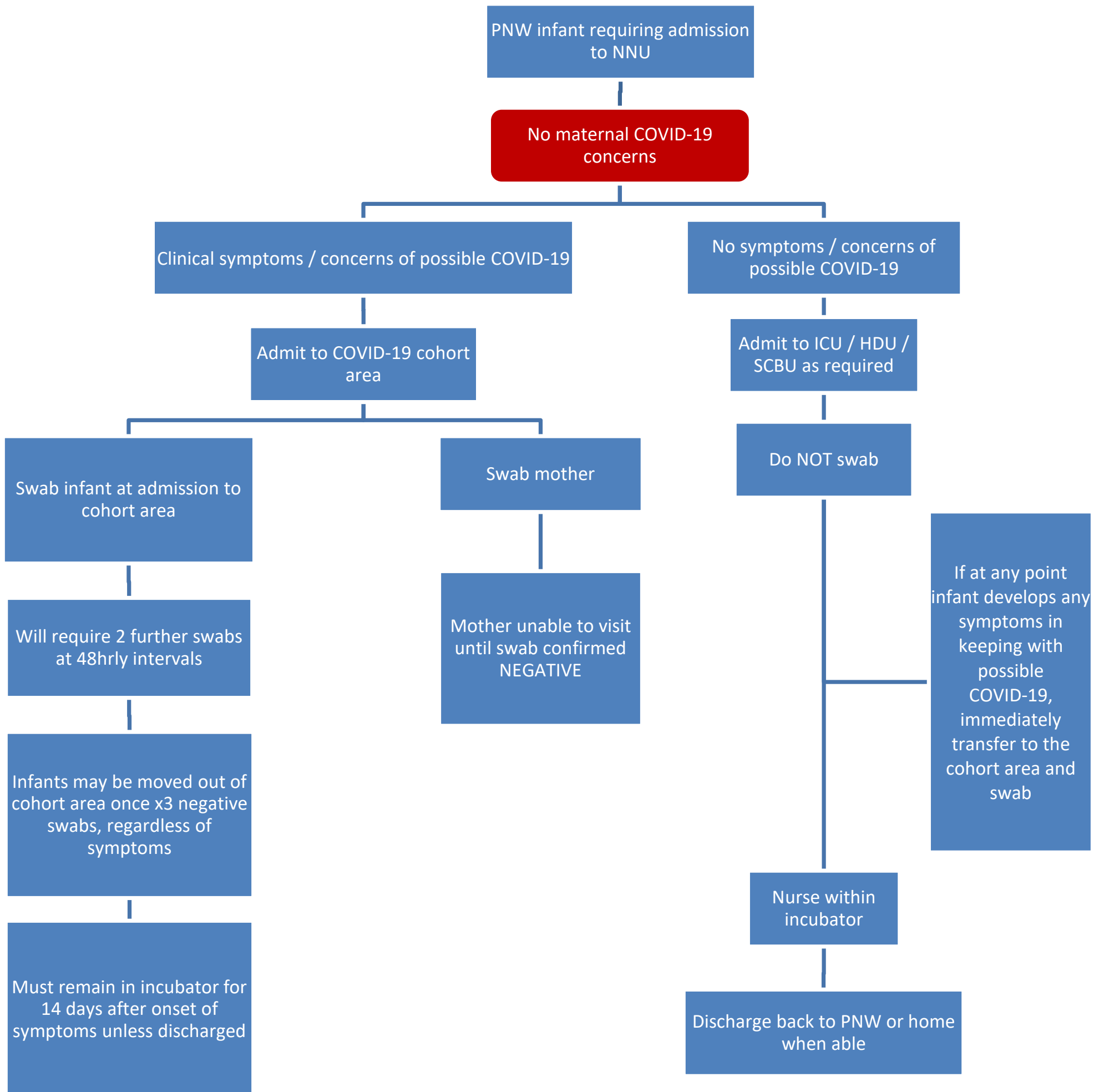




\section{COVID-19 Concerns in NNU Inpatient}

Although strict general IPC protocols remain in place and visiting has been significantly restricted, our vulnerable patients within the NNU remain at theoretical risk of infection with COVID-19 from both parents and staff.

If an infant within the general NNU areas has an unexpected deterioration, COVID-19 should be considered as a differential diagnosis if they fit the 'case definition' as defined by Public Health England:

- They are an inpatient

AND

- have either clinical or radiological evidence of pneumonia OR

- acute respiratory distress syndrome OR

- influenza like illness (fever $\geq 37.8^{\circ} \mathrm{C}$ and at least one of the following respiratory symptoms, which must be of acute onset: persistent cough (with or without sputum), hoarseness, nasal discharge or congestion, shortness of breath, sore throat, wheezing, sneezing

However, the RCPCH have also noted that "newborn infants may not show all the features of an influenza-like illness, particularly a fever, so clinicians should have a high index of suspicion in all infants admitted to NICU and monitor for signs of respiratory illness during the admission".

It remains essential however to consider all other possible pathologies that are encountered within routine neonatal care and there should be a discussion with either the consultant on service or oncall before investigating or cohorting an infant from the general NNU area, for suspected COVID-19.

Below is a flowchart designed to aid with decision making around these infants.

Of note, we again advise that, if an infant is moved to the cohort area and screened for possible COVID-19, the mother should also be swabbed regardless of her symptoms. She will be unable to visit the NNU until her swab is confirmed negative. 


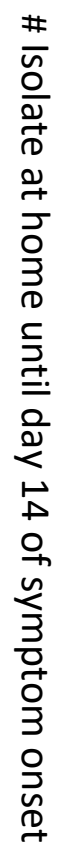
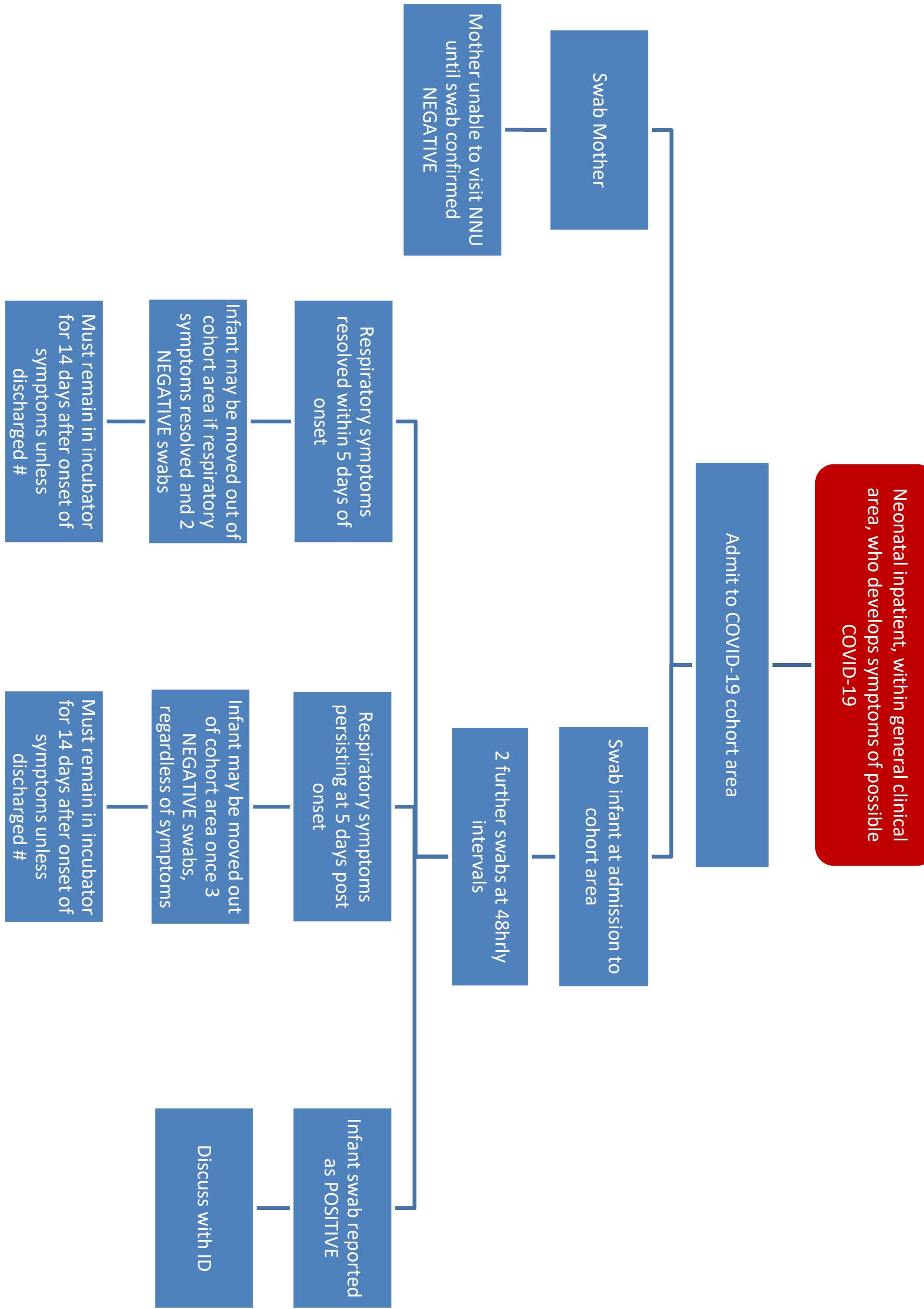
has been created and is situated in the corridor outside the rooms. If the infant requires NNU admission, transport should be via the designated route. If the infant can remain with the mother, PPE must be doffed before leaving.

\section{Working Within The Cohort Area:}

COVID-19 NNU Cohort Areas: With the exception of a facemask (surgical or FFP3), all PPE should be doffed at the door of the infant bays before exiting. The facemask should then be removed just outside the bay before exiting into a Green Zone. Corridors between infant bays are screened off, and foot traffic between these areas is discouraged. Staff will be allocated to work within the cohort area at staff handover. The principle of the minimum number of staff entering infant rooms during a shift should be observed, and records should be kept of all staff entering the cohort area. Staff who are pregnant or have significant chronic health conditions will not be assigned to the cohort area.

\section{When working within the cohort area:}

1. All staff must wear appropriate PPE. Masks, gowns, and hats should be used on a sessional basis. The fluid-resistant surgical mask has a lifespan of approximately 4 hours, with the FFP2 respirators lasting up to 12 hrs unless they become wet. There is no need to change scrubs when out on a break. If an infant within the cohort area is ventilated, closed suction should be used.

2. Due to the risk of contamination, patient notes and observation charts should NOT be kept within the cohort area where possible. If any paper records or charts are used within the cohort area, they must be digitally imaged and archived within the patient record and stored/disposed of once the patient leaves the area. Electronic versions of nursing care notes and observation records have been made available. When possible, the drug Kardex should NOT be used within the room, it should remain outside the cohort room, and drugs should be double-checked with a clean helper where possible. If a Kardex is used within the cohort area, it must be digitally imaged and archived within the patient record and stored/disposed of once the patient leaves the area.

3. The use of reusable equipment should be avoided if possible. If used, it should be decontaminated in the doffing area prior to removal from the cohort area. A dedicated blood gas analyser and ultrasound machine have been made available for use with the cohort area. Blood investigations should be rationalized to a minimum with Point of Care used where possible. If more than one infant within the cohort area requires blood draws, these should be coordinated and performed at the same time if able. Blood samples must be handled and transported to the labs with additional precautions: Perform the blood sample as routine, apply a patient identification label and biohazard sticker to both the sample tube and request form, wipe outside of sample tube with disinfectant, place into a clear leak-proof bag attached to the request form and wipe outside of the bag with disinfectant. Ideally, a buddy should meet you at the door and hold open a second bag for the sample to be placed into. Attach the "COVID-19 biohazard" label to the outside of the second bag, and these must be hand-carried to the lab. No use of the pneumatic tube system is permitted.

Parents are permitted to visit their infant whilst they are in the cohort area, as long as they are COVID-19 suspected or positive but also in adherence to the general restrictions for visiting, as outlined in the visiting policy.

\section{Postnatal Management of the Term, Well Infant}

Term infants who remain well at the time of delivery should remain with their mother even if she is COVID-19 positive, as long as the mother is physically able to care for her child. The pair should be cohorted into an individual room where possible. There is no indication to test the well term infant for COVID-19, regardless of maternal status, unless they become symptomatic.

For mothers who are confirmed COVID-19 positive or suspected, the following measures apply, regardless of the symptoms:

- $\quad$ Strict hand hygiene measures at all times

- $\quad$ The infant should be nursed in an incubator within the room

- $\quad$ Social distancing is observed where possible

- $\quad$ Use of surgical face mask when feeding

- $\quad$ Early discharge of the pair should be considered, with clear handover to the community team

- $\quad$ Staff should wear appropriate PPE when working within the room

- If the mother is acutely unwell and unable to care for her child, the infant should be isolated from the mother, and attempts should be made to identify an alternative non-quarantined caregiver or relative that could provide care for the infant at home.

Term Infants Requiring Review, Investigation and/or Treatment

Routine procedures such as Newborn Hearing Screening and pre-discharge physical examination should be undertaken by midwives and audiology staff as normal. An infant who is cohorted with the mother in an individual room due to maternal proven or suspected coronavirus should have these procedures performed beside the mother, within the individual room.

\section{Management of the Term Infant with Acute Collapse}
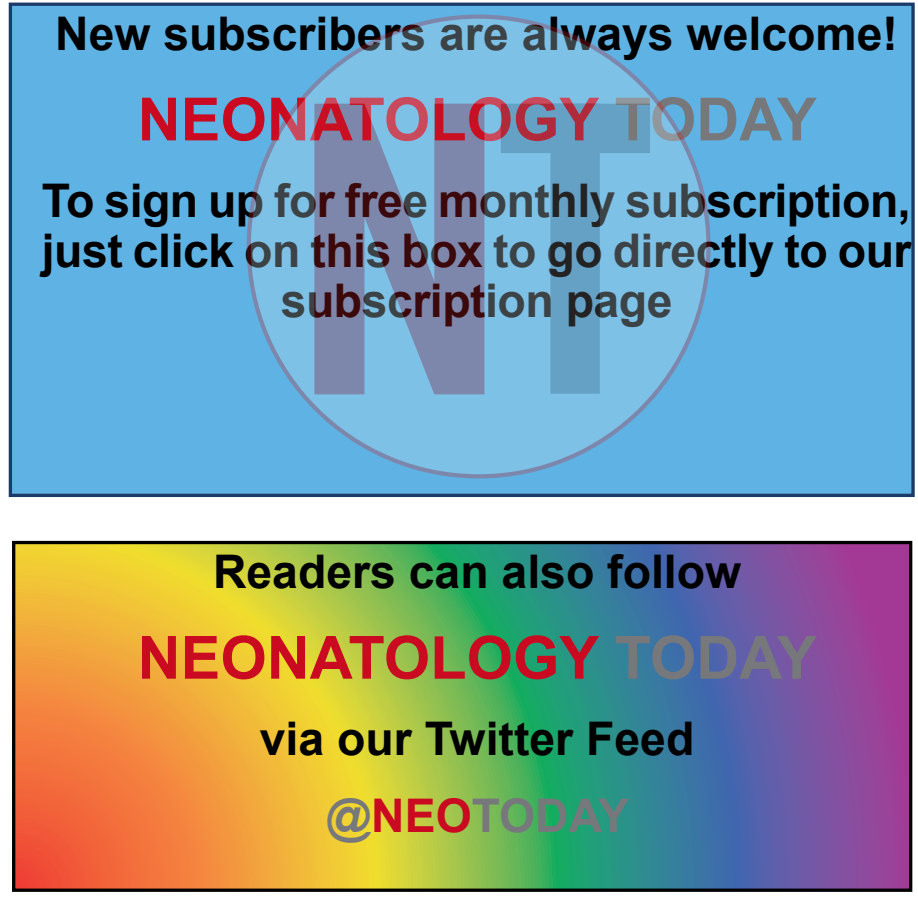
If the neonatal team is called urgently for an acutely unwell infant, the infant will be deemed as potentially infected with COVID-19 regardless of maternal infection status. Given this, it is imperative that staff don full enhanced PPE prior to resuscitating the infant, as your own personal safety is paramount. This is essential, even if it results in a short delay in the initial treatment. Staff will be issued with their own fit-tested FFP3 mask, which should be carried with them while on duty. This will ensure appropriate protection is available for each individual regardless of location. Upon full consideration of available equipment and space within the postnatal areas, we are currently recommending that in event of an acute collapse, the infant should be transferred out of their room or bay and onto the resuscitaire within the ward Treatment Room. While this is not ideal from an infection perspective, it will allow full access to resuscitaire and emergency equipment. Neonatal resuscitation should continue per standard algorithm. Once stabilized, inform the Bed Manager of the pending admission to the NNU should be informed as additional help will be required to ensure a clear route during transfer, and this should be undertaken via designated routes when possible.

\section{Infant Feeding}

Breastmilk and breastfeeding have many significant benefits for mothers and babies. This is particularly true for an infant born prematurely where breast mild is known to help protect against respiratory infections and necrotizing enterocolitis. There is currently no evidence to date that COVID-19 is transferred to breastmilk, and the benefits of breastfeeding are thought to outweigh any potential risks of transmission of coronavirus through breastmilk. Therefore, an infant born to mothers with suspected probably, or confirmed COVID-19 should be fed according to standard infant feeding guidelines, while applying necessary precautions. The main risk for infants breastfeeding is the close contact with the mother and the potential exposure to her infective airborne droplets. It is, therefore important that the benefits of breastfeeding and any potential risks associated with COVID-19 transmission are discussed with the mothers. Infants on the Postnatal Ward or Discharged Home

1. Wash their hands before touching the baby, breast pump or bottles

2. Try and avoid coughing or sneezing on your baby while feeding at the breast

3. Consider wearing a face mask while breastfeeding

4. Where mothers are expressing breastmilk in hospital, a dedicated breast pump should be used

5. Follow recommendations for pump cleaning after each use

6. Consider asking someone else who is well to feed expressed breastmilk

7. For women bottle-feeding with formula or expressed breastmilk adherence to sterilization guidelines is recommended.

\section{Infants within the Neonatal Unit}

For infant admitted to the neonatal unit whose mother is suspected or confirmed as having COVID-19

1. Breastfeeding should be encouraged through supporting mothers who have been separated from their baby to express milk

2. Mothers should have a designated breast pump for exclusive use
3. Specific COVID-19 cleaning procedures should be used when cleansing the breast pump

4. Breastmilk should be expressed, labeled, stored, and transported to the neonatal unit in line with local infection/ COVID-19 control procedures.

\section{Delivery of EBM to NNU from Mothers with Suspected or Confirmed COVID-19}

Unfortunately, parents will not be permitted to visit the unit during the period that their infant is in isolation. However, it is important that maternal milk continues to be used for these infants where possible. Mother's will be given advise as follows:

1. Milk should be labeled and stored at home in the home fridge/freezer until transported

2. Parents should arrange for milk to be transported to the NNU. This needs to be someone who is not requiring isolation for any reason and not from the same household. The milk should be transferred in a cool bag.

3. Staff will meet that person in the reception area. The staff member will wear an apron and gloves to receive the milk. The milk bottles should be transferred from the cool bag into a plastic bag. The NICU staff will take the milk to the storage area and clean with detergent before placing it in the identified area.

4. Extra expressing bottles, labels, and pump attachments should be ordered for collection by phone and picked up at reception when delivering milk.

5. Maternal Expressed breastmilk will be stored in a dedicated COVID 19 fridge/freezer within the NICU.

Of note, when handling this milk at the bedside, staff should continue to comply with current hand hygiene and PPE policy. As an extra precaution, it is advised that staff wipe the outside of bottles/ syringes containing expressed breastmilk with a decontamination wipe before and after any handling.

\section{Visiting Policy}

During these challenging circumstances of the COVID-19 pandemic, visiting across all hospital sites has been prohibited except for exceptional circumstances. Although having a child within neonatal intensive care is a special circumstance, restrictions will apply. Currently, the restrictions are:

1. Visiting is limited to parents or named guardians only

2. Only one parent is allowed at the bedside at a time

3. Siblings are not allowed to visit the unit

4. Parents will not be present on ward rounds

5. Parents or guardians with confirmed or suspected COVID-19 are not permitted to visit the hospital until they are fully recovered and completed the self-isolation period.

6. Parents or guardians in self-isolation are not permitted to visit the hospital

7. In accordance with infectious disease advice, COVID-19 suspected or confirmed mothers should not visit the NNU until symptom-free and at least seven days after the onset of their illness. 
8. In cases where visiting has been restricted, alternatives such as video call may be considered.

\section{Discharge Home and Follow-up}

For COVID-19 suspected or confirmed mothers with a term well infant, the aim is to facilitate early discharge home as soon as is appropriate and safe. These family must self-isolate at home for 14 days following discharge. Several NHS parental information sheets are available on illness in newborns and coronavirus. For infants who have been admitted to the NNU, whose mother is confirmed COVID-19, the need for the family to self-isolate at discharge will depend on the timing of maternal infection relative to the infant's admission. All infants with confirmed COVID-19 infection will require outpatient follow-up, although the timing and duration of this are currently unclear. These infants should be discussed with Infectious Disease consultants regarding appropriate follow-up arrangements prior to discharge.

\section{POLAND}

In Poland, the Ministry of Health assembled national experts in infectious diseases and neonatologists to promulgate guidelines for the management of pregnant women and their infants (13). This group of experts published practice guidelines for managing labor as well as neonatal care guidelines.

\section{The Polish Guidelines are as follows:}

The Instruction sets out the rules for the management of neonates born in the Gynaecology and Obstetrics Research and Teaching Hospital by mothers with a suspected or confirmed diagnosis of infection by, or after contact with, the SARS-CoV-2 coronavirus causing COVID-19.

The Instruction applies to all Hospital Units/Subunits and the Bed Management Department.

The Head of the Hospital Unit/Subunit, the Head Nurse/Midwife, and the head of the organisational unit, are responsible for communicating the contents of the Instruction to their subordinate personnel.

\section{Diagnostic criteria}

Considering that:

1. there is no definite evidence that babies can be infected in the womb, no proof for vertical infection,

2. the effect of the mother's infection in the first and second trimesters of pregnancy on the baby is unclear,

3. there is no conclusive evidence for virus transmission from the mother to the baby during natural childbirth,

the likely route of transmission of the virus from the mother to the baby has been established. Non-compliance with the sanitary regime during childbirth, relating to the mother's failure to use a face mask with filter, increases the risk of neonatal infection with SARS-CoV-2 by the droplet route.

\section{Procedures to follow}

1. The delivery of a neonate by a mother with a suspected or confirmed diagnosis of infection by, or after contact with, the SARS-CoV-2 coronavirus causing COVID-19, requires the appointment of a neonatological team including a physician from the Neonatal Isolation Unit ( $7 \mathrm{am}-3 \mathrm{pm}$ ) and from the Neonatal Unit (after $3 \mathrm{pm}$ and at night). Additional staffing of midwives is decided on by the Head Midwife.

2. The natural birth or caesarean section takes place in a separate isolation zone in the Admission Room. The zone may include the entire Admission Room or a temporary Admission Room for patients with signs of infection. The extension of the isolation zone to encompass the entire Admission Room is done in response to a clinical case (confirmed SARSCoV-2 infection) and a greater number of patients requiring hospitalisation in the GPSK.

3. Depending on the clinical circumstances (suspected or confirmed viral infection in the mother), members of the neonatological team must use personal protective equipment during the delivery, including:

- if the mother shows no symptoms (but she has been in contact with the virus) - barrier gown with long sleeves, safety gloves, face mask with FFP3 or FFP2 filter, safety glasses;

- if the mother shows clinical symptoms and infection with SARS-CoV-2 is confirmed - protective overalls, face mask with FFP3 or FFP2 filter, safety glasses. Protective clothing is available in the Admission Room.

4. If the newborn is isolated from the mother and placed in a closed incubator, members of personnel must wear a barrier gown with long sleeves, safety gloves, a face mask with FFP3 or FFP2 filter, and safety glasses while performing neonatal examinations and providing care.

\section{Neonates not requiring hospitalisation in the NICU}

1. After birth, the newborn remains isolated from the mother in a closed incubator on any premises of the Admission Room other than where the mother is recovering.

2. The newborn does not require hospitalisation for more than two days.

3. While being hospitalised, the newborn can be fed with expressed breast milk, provided that the mother complies with the sanitary regime (face mask with filter, safety gloves, appropriate hand hygiene procedure) - at the neonatologist's discretion.

4. After two days of hospitalisation, the newborn may be discharged and taken home by the legal guardian.

5. At discharge from the hospital, the legal guardian should be instructed to observe the baby for signs of respiratory distress for 14 days after birth.

6. The legal guardian should be instructed to report to the hospital designated by the governor of Wielkopolska Province (Appendix 1) for medical consultation if the baby develops symptoms such as fever, cough, shortness of breath, difficulty breathing, or an increased breathing rate.

7. The legal guardian should be informed about the possibility of calling an emergency medical team to transfer the baby from home to a selected hospital.

8. The neonatologist must notify the District Sanitary Inspector in charge of the territory of the patient with suspected SARSCoV-2 infection (24-hour telephone service: 609794 670).

Neonates not requiring hospitalisation in the NICU, but requiring treatment in a hospital with an infectious diseases unit 
1. After birth, the newborn remains isolated from the mother in a closed incubator on any premises of the Admission Room.

2. The newborn is urgently transferred to a hospital with an infectious diseases unit designated by the Governor of Wielkopolska Province in accordance with Appendix 1 to the Instruction.

3. The patient (in a closed incubator) may be transported to the hospital with an infectious diseases unit by the hospital's neonatal ambulance.

4. The neonatologist must notify the District Sanitary Inspector in charge of the territory of the patient with suspected SARSCoV-2 infection.

Neonates without clinical symptoms of SARS-CoV-2 infection but requiring hospitalisation in the NICU because of their clinical condition after birth

1. After the natural birth or caesarean section, the newborn is placed in a closed incubator and transferred to the Neonatal Isolation Unit.

2. An isolation zone should be designated within the continuous care subunit in the Neonatal Isolation Unit. No other patients may be hospitalised in this zone.

3. While being hospitalised in the isolation zone of the Neonatal Isolation Unit, the baby should be placed in a closed incubator until the isolation is completed (at least 14 days after birth).

4. Taking samples from the baby for identification tests for SARS-CoV-2 should be considered (as set out in the Appendix).

5. Separate personnel should be appointed to care for the baby.

6. For patient care, medical personnel must wear protective clothing: barrier gown with long sleeves, disposable diagnostic gloves, face mask with FFP3 filter, or FFP2 filter in the lock (intermediate space before the isolation zone).

7. After handling the newborn baby, the procedure is to remove gloves first and disinfect the hands. In the lock (intermediate space), the personnel should take off the barrier gown, remove the safety glasses and face mask, and perform an extended hand hygiene procedure (washing and disinfection).

8. The maximum duration for wearing a single mask with a filter is 8 hours and a standard surgical mask -1 hour.

9. As the risk of transmission of infection from the mother to the baby during childbirth is very low, there is no need for the personnel to wear protective overalls.

10. Protective overalls should be worn if the neonatal medical personnel assists in the delivery of a patient with a confirmed infection by SARS-CoV-2, causing COVID-19.

11. The standard hand hygiene procedure should be extended to washing hands with soap and water, followed by disinfection with an alcohol-based antiseptic.

12. The newborn should be isolated for 14 days after birth.

13. During this period, the baby should be fed with a formula.
14. The isolation zone should be provided with the necessary equipment for the treatment and care of the isolated newborn. The equipment must remain in the zone for the entire period of isolation.

15. Waste and bedding must remain in the lock of the insulation zone until being collected directly by the cleaning services (laundry staff, waste transport workers) according to the daily schedule.

16. Hospital bedding must be placed securely in a red plastic bag and marked with a black marker: CAUTION - CONTAMINATED BEDDING, SUSPECTED SARS-CoV-2 INFECTION.

17. The cleaning of the isolation zone is carried out according to the cleaning company's procedures approved by the hospital representative. The person responsible for overseeing the proper conduct of the procedure is the Specialist for Epidemiology.

18. After the end of the isolation period, it is advisable to have the premises disinfected by fogging, subject to arrangements with the unit management personnel. Responsible Person: Specialist for Epidemiology.

19. Any questions or concerns should be directed to the Specialist for Epidemiology

There have been no critical analyses of whether adherence to the guidelines published in Ontario, Canada, the American Academy of Pediatrics, Ireland, Northern Ireland or Poland have been thoroughly implemented by all maternity and infant care units. Such an international survey would permit an analysis of different approaches and their effectiveness in mitigating the transmission of COVID-19, and establish if these approaches are unnecessarily restricting the support of mothers during labor and delivery interfering with attachment and feeding of her infant. Furthermore, ideal methods for ongoing infant and mother evaluation and care during the 4th trimester after birth evaluating growth, physical development, and providing recommended immunizations between 6-8 weeks after birth are still under development.

Based on empirical evidence through May 2020, vertical transmission of the virus through the placenta, amniotic fluid, or breastmilk has not been demonstrated. Further, neonatal mortality rates specific to COVID-19 are minimal with reports from China, Italy, and one two-center report from New York City $(14,15)$. These reports to date have been of limited numbers, and have shown variation in mother-infant dyad separation and postpartum practices between reporting centers. Vertical transmission cannot be ruled out. Horizontal transmission from mother to infant may occur, thus increasing exposure risk to care personnel caring for the newborn. Sutton and coworkers reported from New York City that universal screening of 215 women presenting to labor and delivery units, $4(1.9 \%)$ of whom has a fever or other symptoms of COVID-19 and all 4 tested positive, while of 211 without symptoms on admission, $29(13.7 \%)$ were found to be positive for COVID-19 but asymptomatic at presentation. Of these, three women developed a fever prior to hospital discharge (mean time two days), and two were treated with antibiotics for presumptive endomyometritis. One patient was felt to have COVID-19 received supportive care. (16) These authors suggest that universal testing of all women admitted to Labor and Delivery suites is beneficial better, informs healthcare providers of appropriate isolation procedures and bed assignments, informs neonatal care providers, and more appropriate allocation of PPE, and thus lessening transmission of the virus. In Southern Connecticut, 782 women presenting for childbirth were screened for COVID-19; $1.5 \%$ were previously diag- 
nosed with COVID, and of the remaining 770 women $30 /$ of 770 (3.9) tested positive for COVID-19 of whom $73.3 \%$ were without symptoms. The overall prevalence of positive test results among asymptomatic women was $2.9 \%$ to $5 \%$ over the month of testing. No asymptomatic women who tested negative developed symptoms during their hospital stay and no healthcare workers were removed from work due to SARS-CoV-2 exposure or transmission from a known positive mother via patient contact (17)

Neonatologists must also be concerned with late-onset sepsis associated with COVID-19. As reported in addition to this issue, and Munoz et al. from Houston (18) who reported on a three-week-old infant and born at 36 weeks gestation with a 2-day history of nasal congestion, tachypnea, and reduced feeding who had received a 48-hour course of antibiotics for suspected sepsis but in whom bacterial cultures were negative. The initial chest radiograph showed bilateral linear opacities and consolidation in the right upper lobe. Oxygen was administered, and ampicillin and gentamicin were initiated. After transfer to a children's hospital, he required intubation, volume support, and vasopressors. Reverse-transcription PCR testing to detect SARS-VOV-2 on admission to the children's hospital was positive by day seven, and he was treated and discharged on day 9 . Only one of eight household contacts was symptomatic, but none were tested for SARS-CoV-2. In the May issue of Neonatology, Liu and Stovall (19) reported a 33week infant whose mother on day 6 had a syncopal like episode while visiting her infant but did not have symptoms consistent with COVID-19, and she continued to visit her baby. On the infant's 19th day of life, the mother was hospitalized for cough, fever, and hyperglycemia and was found to be SARS-CoV-2 PCR positive. The infant was weaned from expressed maternal milk, placed on premature formula, and placed in a negative pressure room. On the infant's 22nd day of life, the infant's SARS-Cov-3 PCR was positive. On days of life 23 through 25, the infant had mild nasal congestion, occasional sneezing, and cough. Contact tracing was rigorous, and 74 potential exposures were documented; however, secondary exposure risk to healthcare personnel was deemed low exposure to the mother within 6 feet for $>5$ minutes with contact through the portals of the incubator with the mother holding and feeding without either a mask or gown. Buonsenso et al. reported outcomes, seven pregnant women, with documented SAR-CoV-2 infection, one woman had a spontaneous abortion at eight weeks gestational age, four women recovered and continues to be followed, and of the two newborns delivered both were negative at birth, but one was found to SARS-COV-2 positive at eight days. (20) Thus, the horizontal transmission was strongly inferred. These cases suggest that infants presenting with a clinical presentation consistent with late-onset neonatal sepsis require an expanded search for pathogens, including the SARS-Cov02 test by PCR.

According to the aforementioned guidelines, taking simple precautions such as assigning designated staff for attending deliveries (if intervention is anticipated), allowing rooming-in of the baby with its mother and monitoring breastfeeding while keeping appropriate physical barrier, staff adherence to the appropriate PPE policy, and facilitating respiratory support inside incubators (if needed) would allow safe management of those suspected cases. This would apply to ether infants of asymptomatic mothers with a history of significant COVID-19 contact or infants of symptomatic mothers awaiting COVID-19 test results. It is clear that additional resources are needed, including increased physical distance and, moreover, PPE not only for healthcare workers but also for parent(s) visiting one at a time for short intervals, and rapid testing with acceptable sensitivity and specificity need to be made available to neonatologists. Also, additional incubators must be available to safely care, ideally in a negative pressure environment, for the medical needs of mothers and their babies, and for the health care workers to be able to care for them safely.
In conclusion, the main threats presented by COVID-19 to the safe provision of maternity care are related to the prevalence of the condition in the community and to the possibility that asymptomatic carriers could expose other mothers or hospital staff to infection. Large scale staff reductions due to the need for isolation or due to infection will reduce the safety and efficiency of maternity care provision. Even in countries with high prevalence and high numbers of fatalities, serious illness in mothers seems rare, and seriously affected infants are thankfully extremely rare. This may be due to effective self-isolation being practised by a vast majority of expectant mothers. As "stay at home" or "lockdowns" ease in the coming months, the pressure to reduce visitor restrictions will increase, with an inevitable increase in risk to staff. The staff will need to be vigilant regarding the risk which mothers and their partners present and will need to wear appropriate PPE to mitigate this risk. More importantly, they need to be aware of the risk they present to each other and to ensure adequate hand hygiene and social distancing to avoid congested communal staff facilities such as rest areas, and dining areas, and to be aware of the surface transmission risks presented by shared computers and keyboards. Team building and educational activities have rapidly migrated to electronic platforms, and these enhanced efforts will need to be sustained for the foreseeable future. For the present, and probably for months, there will be a reversion to what has been "normal practice."

As in North America and "across the pond," we do indeed live in challenging times.

\section{References:}

1. World Health Organization. (April 22, 2020) What matters to women in the postnatal period? http://www.who.int/news-room/detail/22-042020-what-matters-to-women-in-the-postnatal-period.

2. U.S. Department of Health and Human Services, Centers for Disease Control and Prevention (April 4, 2020) Considerations for inpatient obstetric healthcare settings. https://cdc.gov/coronavirus/2019-ncov/hcp/inpatientobstetric-healthcare-guidance.html.

3. Puopolo, KM, Hudak ML, Kimberlin DW, Cummings J. Initial Guidance: Management of Infants Born to Mothers with COVID-19: April 2, 2020, American Academy of Pediatrics

4. Ma X, Zhu, J, Du, Uzhong. Neonatal Management During the Coronavirus Disease (COVID-19) Outbreak: The Chinese Experience. NeoReview May 2020 , 21(5) e293-2297.

5. Ontario Health Toronto: Toronto Region COVID-19 Hospital Operations Table Version Date March 17, 2020, taken from Graham R. Covid-19 and he NICU Balancing Safety and Care. Neonatology Today, April 2020. 15(4): 25-28.

6. Trevisanuto D, Moschino L, Doglioni N, Roehr, CC, Gervasi MT, Baraldi E. Neonatal Resuscitation Where the Mother Has a Suspected or Confirmed Novel Coronavirus (SARSCoV-2) Infection: Suggestion for a Pragmatic Action Plan. Neonatology April 24, 2020, DOI 10.1159/000507935.

7. $\mathrm{Ng}, \mathrm{PC}$. Infection control measures for COVID-19 in labour suite and neonatal unit-A commentary Neonatology 2020 DOI:10.1159/000508002

8. American Heart Association. Interim Guidance for Basic and Advanced Life Support in Children and Neonates with suspected or confirmed COVID-19. Pediatrics 2020 doi: 10.1542/peds. 2020-1403

9. Zhu H, Wang L, Fang $C$, et al. Clinical analysis of 10 neonates born to mothers with 2019-nCOV pneumonia. Transl. Pediatr. 2020; 9(1):51-60 dol: 10.21037/tp.2020.02.06

10. Chen H. Guo J, Wang $C$ et al. Clinical characteristics and intrauterine vertical transmission potential of COVID-19 infection in nine pregnant women: a retrospective review of medical records. Lancet 2020; 396(10226):809-815 dol:10.1016/S0140-673(20)30360-3. 
11. Royal College of Physicians and Surgeons, Ireland: Institute of Obstetricians and Gynaecologists of the Royal College of Physicians of Ireland outlined recommendations for Neonatal Management for Maternal and Neonatal Management, April 2020.

12. Walker A. Royal Maternity Hospital Neonatal Unit COVID-19 Policy., Edition April 5 29, 2020. Belfast Health and Social Care Trust, Belfast, Northern Ireland.

13. Ziarnik M, Sosnowska J, Chmaj-Wierzhowska K, Figlerowicz M, Wysocki J, Mazela J. COVID-19 -epidemiologia, obraz kliniczyny oraz postepowanie z ciezarna I noworodkiem. Standary Medyczne Pediatria/April, 2020.

14. Zeng L, Xia S, Yuan W. et al. Neonatal Early-Onset infection with SARS-CoV-2 in 33 neonates born to mothers(https// www. statista.com/statistics/1043366/novel-coronavirsu2019nvov-cases-worldwidebycounty.html) rs with COVID-19 in Wuhan, China. JAMA Pediatr. March 26, 2020 (online) doi:10.1001/jamapediatrics.2020.0878.

15. Breslin N, Baptiste C, Gyamfi-Bannerman C, Miller $R$. Martinez R, Bernstein K, Goffman D. COVID-19 infection among asymptomatic and symptomatic pregnant Women: Two weeks of confirmed presentation to an affiliated pair of New York City hospitals. American J. Obstet \& Gynceol MFM. Advance online publication. Doi: 10.1016/ ajogmf.2020.100118.

16. Sutton D, Fuchs K, D'alton M, Goffman D. Universal screening for SARS-CoV-2 in Women Admitted for Delivery. N Engl J. Med 2020 382: 2163. Doi: 10.1056/NEJMc2009316.

17. Campbell, KH, Tornatore JM, Lawrence et al. Prevalence of SARS-CoV-2 Among Patients Admitted for Childbirth in Southern Connecticut. JAMA May 26.2020. doi:10.1001/ jama2020.8904.

18. Munoz AC, Nawaratne U, McMann D, Ellsworth M, Meliones J, Boukas K. NEJM April 22, 2020: $382 . e 49$ doi: 10.1056/ NEJMc2020614.

19. Liu W and Stovall S. Case of a preterm newborn with the nosocomial Acquisition of COVID 19 Infection in the Neonatal Intensive Care Unit and Contract Tracing. Neonatology Today 15(5) May 2020: 12-20.

20. Buonsenso D, Costa S, Sanguinetti $M$ et al. Neonatal Late COVID-19 Infection with Severe Acute Respiratory Syndrome Coronavirus 2. Am J Perinatology 2020 doi: 10.1055/s-9949-1710541.

21. Note added in proof: On May 20, 2020, the Centers for Disease Control and Prevention Revised their recommendations on "Evaluation and Management Considerations for Neonates At Risk for COVID-19 and Care for Breastfeeding Women for the USA.

Disclosures: The authors do not have any disclosures.

The authors wish to acknowledge the special assistance of Thomas A. Clarke, MD, Retired Consultant, Rotunda Hospital, Dublin, Ireland, Co-Editor

NT

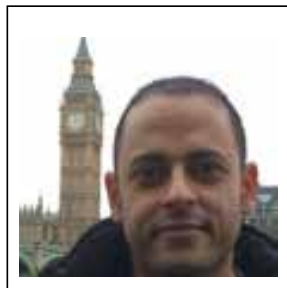

Ahmed Afifi, MBBCH, MSc Neonatal/Paediatric Registrar, Rotunda Hospital,

Dublin, Ireland

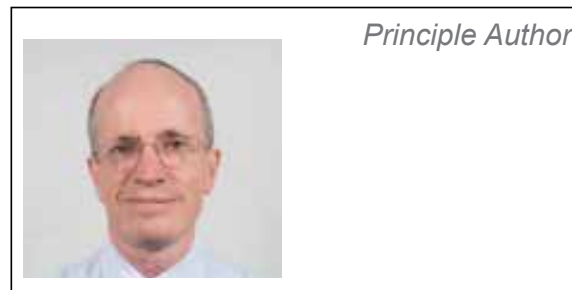

David Corcoran MD FRCPI IMC 9632

Consultant Neonatologist and Paediatrician Clinical Associate Professor of Paediatrics

Rotunda Hospital,

Royal College of Surgeons in Ireland

Dublin, Ireland

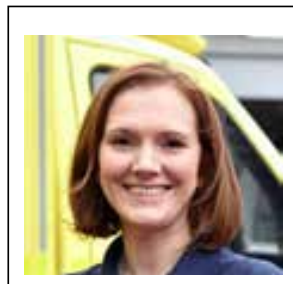

Allison C. Walker, MD,

Consultant Neonatologist,

Royal Maternal Neonatal Unit

Belfast, Northern Ireland 


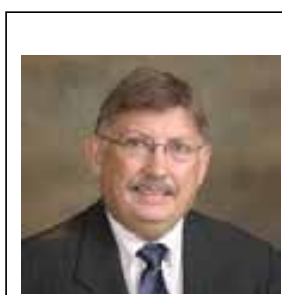

Corresponding Author

T.Allen Merritt, MD

Professor of Pediatrics

Loma Linda University School of Medicine

Division of Neonatology

Department of Pediatrics

email: T. Allen Merritt < allenmerritt.md@gmail.com>

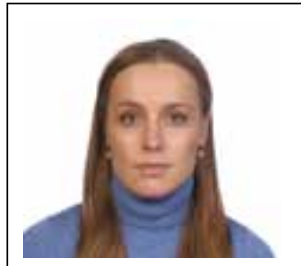

Aleksandra M. Adamczak, MD

Neonatology Fellow, PGY1

Cook County Health

Chicago, IL

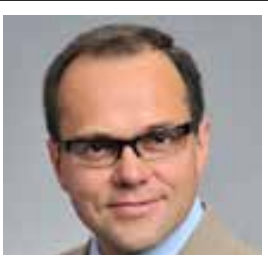

Jan Mazela, M.D., Ph.D.

Professor of Paediatrics,

Poznan University of Medical Sciences,

Poznan, Poland

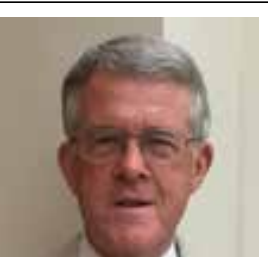

Thomas A Clarke, MD

Emeritus Consultant in Neonatology

The Rotunda Hospital,

Dublin. Ireland
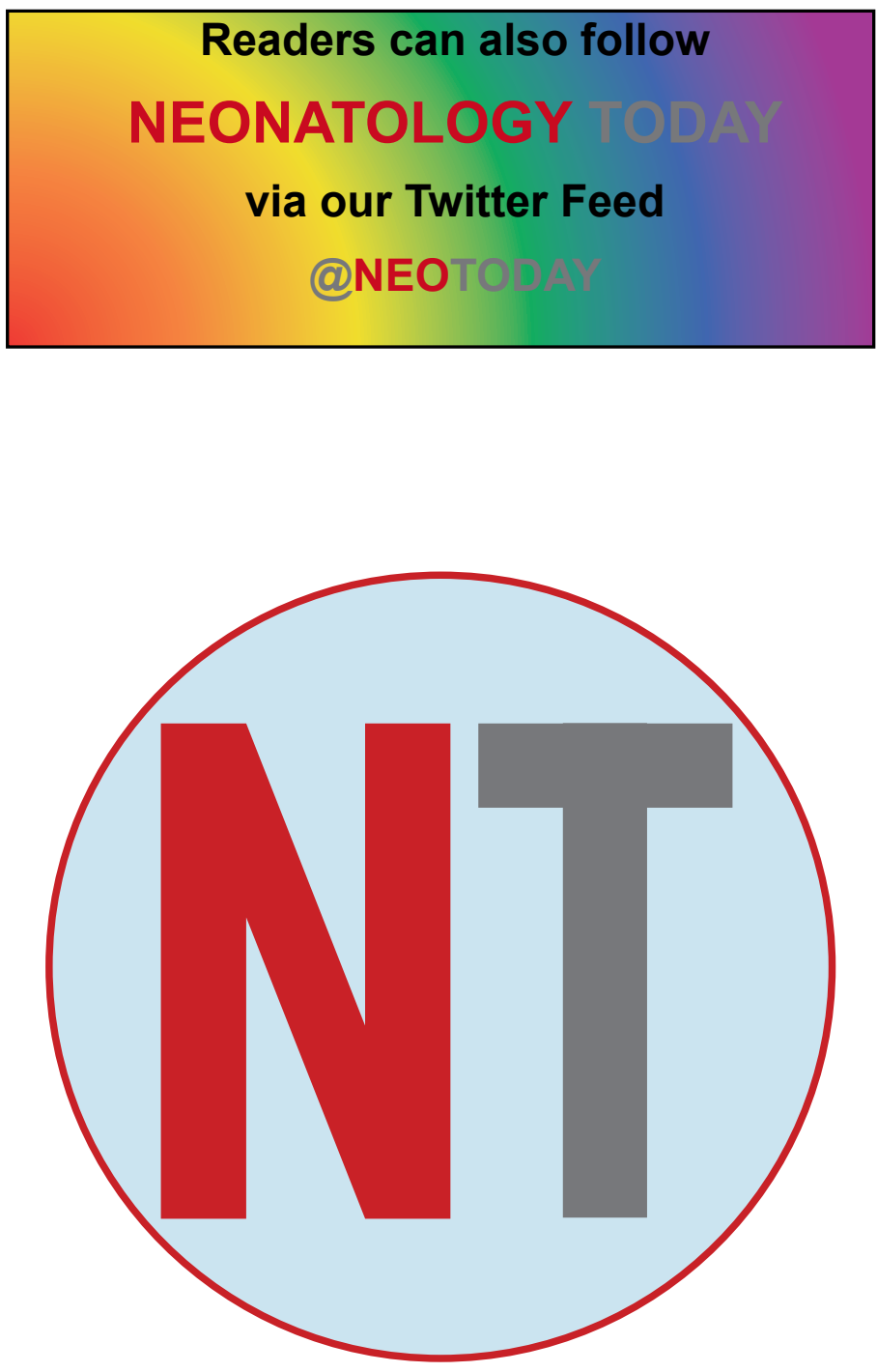